九州大学学術情報リポジトリ

Kyushu University Institutional Repository

Diagnosis of Mungbean (vigna radiate)

Bradyrhizobia Isolated from Kyushu Island of Japan Based on Whole Cellular Fatty Acid Analys is

Matsumoto, Masaru

Institute of Tropical Agriculture, Kyushu University

https://doi.org/10.5109/4662

出版情報: 九州大学大学院農学研究院紀要. 50 (2)，pp.489-496，2005-10-01. Faculty of Agriculture, Kyushu University

バージョン：

権利関係 : 
J. Fac. Agr., Kyushu Univ., 50 (2), 489-496 (2005)

\title{
Diagnosis of Mungbean (Vigna radiate) Bradyrhizobia Isolated from Kyushu Island of Japan Based on Whole Cellular Fatty Acid Analysis
}

\author{
Masaru MATSUMOTO \\ Institute of Tropical Agriculture, Kyushu University, Fukuoka, 812-8581, Japan \\ (Received June 16, 2005 and accepted July 26, 2005)
}

\begin{abstract}
Previous studies have already reported that cellular fatty acid analysis is a useful taxonomic tool for classifying and identifying Bradyrhizobium strains. In this study, the fatty acid profiles of 13 strains of MAFF collections and 36 strains collected from Fukuoka, Saga, Oita, Kumamoto and Kagoshima Prefecture belonging to unknown species of Bradyrhizobium were evaluated. Total 11 fatty acids were identified and qualitative and quantitative variations of fatty acid compositions were observed. Data of fatty acid compositions obtained from each strain was statistically investigated by PCA and PCGMA clustering. Statistical analysis showed that the dominant five distinct clusters were formed and conveniently grouped as FAG-I to FAG-V. FAG-I and FAG-II strains were closely relationships to the strain of $B$. elkanii. FAG-III and FAG-V strains were relevant to the strain of $B$. japonicum. On the other hand, FAG-IV strains isolated from mungbean root nodules were clustered with $B$. japonicum FAG-III and FAG-V but obviously different compositions of fatty acid and distinguishable to these two subgroups.
\end{abstract}

\section{INTRODUCTION}

The bacteria that form nodules on leguminous plants and fix atmospheric nitrogen are currently classified into three genera: Rhizobium, Azorhizobium and Bradyrhizobium. The genus Bradyrhizobium was recognized as the slow-growing members of the genus Rhizobium (Jordan 1994). The taxonomy of the Bradyrhizobium, B. japonicum (Hollis et al., 1981) and B. elkanii (Kuykendall et al., 1992) were established species. Recent research have proposed a new species, $B$. liaoningense was an extra-slow growing member and characterized as unique phenotype and genotype (Xu et al., 1995). Moreover, Bradyrhizobium sp. isolated from the root nodules of peanut (Arachis hypogaea) was also performed on the study (Zhang et al., 1999).

On the other hand, miscellaneous rot-nodule bacteria belonging to the genus Bradyrhizobium have been reported. Phenotypic and genotypic characterization of slow-growing bradyrhizobia obtained and isolated from Acacia albida (Dupuy et al., 1994), Aeschynomene (So et al., 1994), Arachis hypogaea (van Rossum et al., 1995; Urts and Elkan 1996), Centrosema and Desmondium (Gao et al., 1994) and Lupinus (Bottomley et al., 1994) suggested the existence of additional taxonomic variations in the genus Bradyrhizobium. These genus Bradyrhizobium is now aspired to rapid and easy identification at the species revel because of changeable of taxonomy and possibility to additional species. However, phenotypic and genotypic characteristics of genus Bradyrhizobium obtained from Vigna radiate were not fully understood at a species and taxonomical revels. 
Previous research showed that whole cellular fatty acid analysis was successfully characterized and evaluated the Bradyrhizobium strains, showing the existence of different phenotypic variations of two subgroups in $B$. japonicum, two subgroups in $B$. elkanii and one subgroups of Bradyrhizobium sp (Jarvis and Tighe 1994; Tighe et al., 2000; Graham et al., 1995). This analysis is based on the extraction of fatty acid-methyl ester (FAME) from bacterial cells, determination of the cellular fatty acid compositions and comparison of the fatty acid profiles among strains.

In this study, we characterized the phenotypic differentiation of Bradyrhizobium obtained from the root nodule of mungbean (Vigna radiata) in Kyushu area of Japan using whole cellular fatty acid analysis.

\section{MATERIALS AND METHODS}

\section{Bacterial strains}

The strains of Bradyrhizobium used in this experiment are listed in Table 1. They include seven strains of B. japonicum, six strains of $B$. elkanii and 36 strains of unknown species of Bradyrhizobium isolated from root nodule of mungbean. As a control, one isolates of Rhizobium leguminosarum was also used in this experiment. Mungbean was grown on paddy field soils of winter season after finishing harvest of rice crops that were Fukuoka, Oita, Saga, Kumamoto and Kagoshima Prefecture in Japan. Strains were maintained routinely as freeze-dried ampoules and subcultured onto east extract mannitol agar to confirm colony characteristics. Cultivation prior to whole cellular fatty acid analysis was as reported by Javis and Tighe (1994) with slight modification by 7 days incubation periods were needed to achieve satisfactory cell mass.

\section{Isolation and Identification of Bradyrhizobium strains from mungbean root nodules}

Isolation of Bradyrhizobium strains used in this experiment was according to the method of Somasegaram and Hoben (1994). Strains of B. japonicum and B. elkanii identified by this experiment were confirmed using RFLP analysis based on PCR amplified nod genes described by Yokoyama et al. (1996).

\section{Whole cellular fatty acid analysis}

Harvesting and extraction procedure of saponification, methylation and recovery of fatty acids were performed according to the method described by Sassor (1990) slight modifications. Analysis of FAME was performed by a Shimadzu GC-17A gas chromatograph equipped with a HR-SS-10 capillary column. Comparison of similarity data of fatty acid profiles were accomplished by the two statistical methods, principal component analysis (PCA) and cluster analysis based on the detected whole cellular fatty acid compositions using the SYSTAT software. Each cluster was combined with unweighted pair-group method with arithmetic average (UPGMA) by comparing Euclidean distance.

The fatty acid compounds for each strain were identified by first converting the total cellular fatty acids to FAME. Cellular fatty acids were identified by comparing the equivalent chain length (ECL) of each compound to a retention time that represents the manufactured fatty acid compounds. The quality of each compound in a strain was determined 
Table 1. Lists of strains of Bradyrhizobium used in this experiment.

\begin{tabular}{|c|c|c|c|}
\hline Species & Strain & Origin & Host \\
\hline \multirow[t]{7}{*}{ B. japonicum } & MAFF303138 & Kumamoto & Soybean \\
\hline & MAFF303154 & Kumamoto & Soybean \\
\hline & MAFF303174 & Kumamoto & Soybean \\
\hline & MAFF303178 & Fukuoka & Soybean \\
\hline & MAFF303166 & Nagasaki & Soybean \\
\hline & MAFF303195 & Kagoshima & Soybean \\
\hline & MAFF303199 & Kagoshima & Soybean \\
\hline \multirow[t]{6}{*}{ B. elkanii } & MAFF303126 & Wakayama & Soybean \\
\hline & MAFF303142 & Tottori & Soybean \\
\hline & MAFF303168 & Kagawa & Soybean \\
\hline & MAFF303193 & Niigata & Soybean \\
\hline & MAFF303196 & Kagoshima & Soybean \\
\hline & MAFF303203 & Kagoshima & Soybean \\
\hline \multirow[t]{36}{*}{ Bradyrhizobium sp. } & MAFF210213 & Ibaraki & Mungbean \\
\hline & MAFF210214 & Ibaraki & Mungbean \\
\hline & KU-T12 & Fukuoka & Mungbean \\
\hline & KU-T24 & Fukuoka & Mungbean \\
\hline & KNS-331 & Fukuoka & Mungbean \\
\hline & KNS-279 & Fukuoka & Mungbean \\
\hline & KNS-411 & Fukuoka & Mungbean \\
\hline & FK7-5 & Fukuoka & Mungbean \\
\hline & FK-8-3 & Fukuoka & Mungbean \\
\hline & SEB7-8 & Fukuoka & Mungbean \\
\hline & HTA-01 & Oita & Mungbean \\
\hline & HTA-02 & Oita & Mungbean \\
\hline & HTA-03 & Oita & Mungbean \\
\hline & HTA-04 & Oita & Mungbean \\
\hline & HTA-05 & Oita & Mungbean \\
\hline & SGU-1T & Saga & Mungbean \\
\hline & SGU-2T & Saga & Mungbean \\
\hline & SGU-11T & Saga & Mungbean \\
\hline & SGU-3T & Saga & Mungbean \\
\hline & SGU-4T & Saga & Mungbean \\
\hline & SGU-31T & Saga & Mungbean \\
\hline & KIC-21 & Kumamoto & Mungbean \\
\hline & KIC-55 & Kumamoto & Mungbean \\
\hline & KIC-64 & Kumamoto & Mungbean \\
\hline & KIC-73 & Kumamoto & Mungbean \\
\hline & $\mathrm{KIC}-83$ & Kumamoto & Mungbean \\
\hline & HT-3 & Kumamoto & Mungbean \\
\hline & HT-7 & Kumamoto & Mungbean \\
\hline & $\mathrm{HT}-11$ & Kumamoto & Mungbean \\
\hline & 27-IB1 & Kagoshima & Mungbean \\
\hline & 27-IB2 & Kagoshima & Mungbean \\
\hline & 32-IB4 & Kagoshima & Mungbean \\
\hline & 33-IB6 & Kagoshima & Mungbean \\
\hline & 18-IB8 & Kagoshima & Mungbean \\
\hline & MK-03-1 & Kagoshima & Mungbean \\
\hline & MK-03-5 & Kagoshima & Mungbean \\
\hline Rhizobium leguminosarum & Quit-4FK & - & Soybean \\
\hline
\end{tabular}


as a percentage of the total amount of fatty acid compounds present for that strain. Nomenclature of each fatty acid used in this experiment was according to the notation described by Tighe et al. (2000).

\section{RESULTS}

\section{Fatty acid compositions}

Fatty acid components detected in identified strains contained 16:0, 16:1 $\omega$ 7cis, 18:0 and summed feature 7. Additional fatty acids contained individual species included 16:1 $\omega 5$ cis, 17:0, 17:0 cyclo, 17:1 $\omega 6$ cis, 17:1 $\omega 8$ cis, 19:0 cyclo $\omega 8$ cis and 20:0 $\omega 6,9 \mathrm{cis}$. In this experiment, qualitative and qualitative variation of fatty acid compositions were also contained in identified strains of B. japonicum and B. elkanii (Table 2.).

Fatty acid components of unknown Bradyrhizobium species were obtained 11 fatty acids, representing 16:0, 16:1 $\omega 5$ cis, 16:1 $\omega 7$ cis, 17:0, 17:0 cyclo, 17:1 $\omega 6$ cis, 17:1 $\omega$ $8 \mathrm{cis}, 18: 0,19: 0$ cyclo $\omega 8 \mathrm{cis}, 20: 0 \omega 6,9 \mathrm{cis}$ and summed feature 7 . Examination of unknown Bradyrhizobium species indicated that qualitative and quantitative variations of fatty acid compositions were observed at the fatty acids of 16:0, 16:1 $\omega 5$ cis, 19 cyclo $\omega 8$ cis and summed feature 7 (Table 2.).

Table 2. Differences in mean fatty acid composition of five groups of Bradyrhizobium strains.

\begin{tabular}{|c|c|c|c|c|c|}
\hline \multirow{2}{*}{ Fatty acid } & B. elkanii & B. elkanii & B. japonicum & Bradyrhizobium sp. & B. japonicum \\
\hline & FAG-I & FAG-II & FAG-III & FAG-IV & FAG-V \\
\hline $16: 0$ & 11.92 & 9.77 & 11.74 & 12.17 & 12.38 \\
\hline $16: 1 \omega 5 \mathrm{cis}$ & nd & nd & nd & 2.86 & 2.78 \\
\hline $16: 1 \omega 7$ cis & 0.50 & 0.52 & 0.96 & 0.79 & 0.88 \\
\hline $17: 0$ & nd & nd & 0.14 & 0.19 & nd \\
\hline 17:0 cyclo & 0.97 & 0.53 & nd & nd & nd \\
\hline $17: 1 \omega 6 \mathrm{cis}$ & nd & nd & 0.15 & nd & 0.14 \\
\hline $17: 1 \omega 8 \mathrm{cis}$ & nd & nd & nd & 0.44 & 0.46 \\
\hline $18: 0$ & 0.35 & 0.14 & 0.54 & 0.60 & 0.72 \\
\hline 19:0 cyclow $8 \mathrm{cis}$ & 19.12 & 8.67 & 1.66 & 4.56 & nd \\
\hline $20: 2 \omega 6,9$ cis & 0.28 & nd & nd & nd & nd \\
\hline Summed feature 7 & 66.86 & 80.38 & 84.81 & 78.39 & 82.64 \\
\hline
\end{tabular}

Note: Figures in parentheses indicate the standard deviation. nd, values less than $0.01 \%$ of total or in which the standard deviation exceeds the mean.

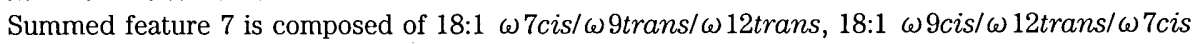
and 18:1 $\omega$ trans/ $\omega 9$ trans/ $\omega 7$ cis fatty acids.

\section{Clustering analysis}

On the basis of FAME analysis and UPGMA clustering, the 36 strains of unidentified Bradyrhizobium, seven strains of B. japonicum and six strains of $B$. elkanii examined in this experiment fell into five distinct groups (Fig. 1.). The FAME profiles of each group are shown in Fig. 1. and Table 2. These included one cluster (FAG-I) that included three strains (MAFF303193, MAFF303203 and MAFF303196) previously identified as $B$. 
Euclidean Distance

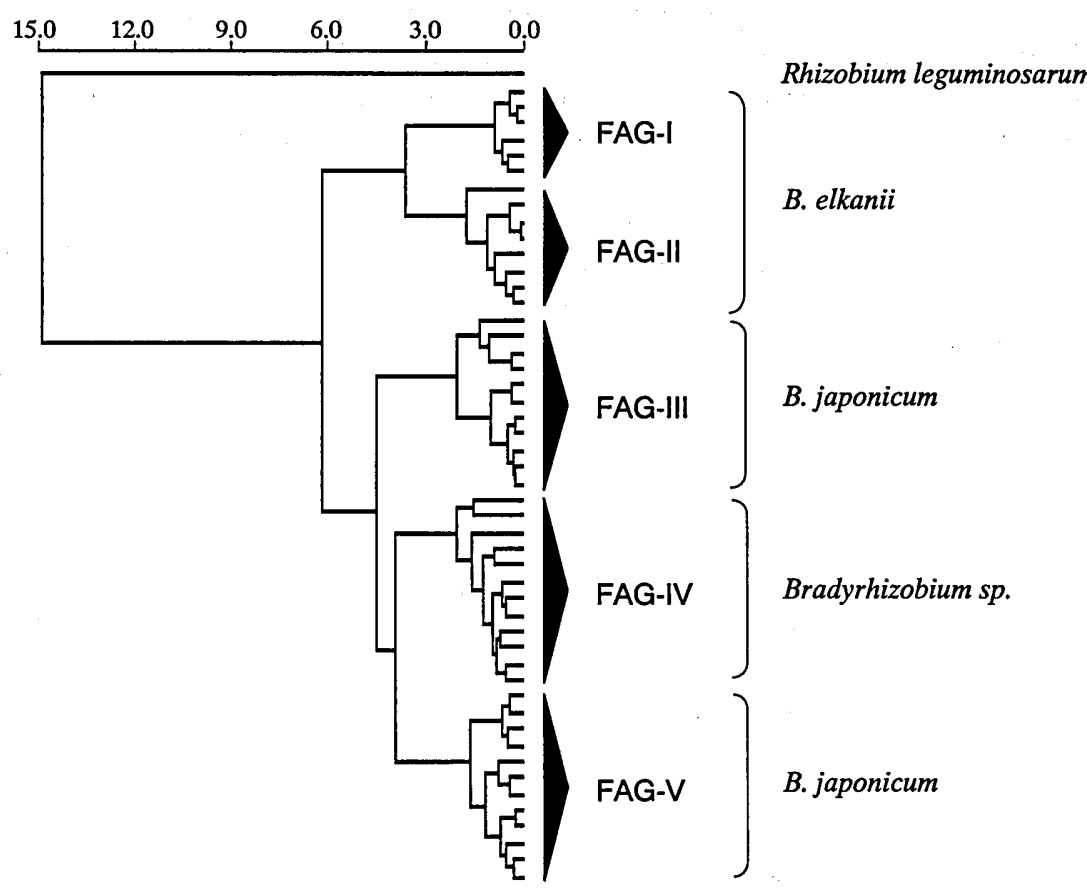

Fig. 1. Simplified UPGMA dendrogram showing the relationships among 49 Bradyrhizobium strains based on total fatty acid (FAME) analysis. Clusters defined in this figure contain 7 strains of $B$. japonicum, 6 strains of B. elkanii and 36 strains of Bradyrhizobium sp. isolated from mungbean root nodules, respectively.

elkanii, and another cluster (FAG-II) that contained three strains (MAFF303142, MAFF303168 and MAFF303126) also identified as B. elkanii. FAME profiles of these strains of $B$. elkanii were characterized by the quantitative variations in 20:0 $\omega 6,9$ cis and qualitative variations in 16:0, 19:0 cyclo $\omega 8$ cis and summed feature 7 . The other clusters included one cluster (FAG-III) that included three strains (MAFF303199, MAFF303166 and MAFF303195) previously identified as B. japonicum, and other cluster (FAG-V) contained four strains (MAFF303178, MAFF303174, MAFF303154 and MAFF303138) also identified as B. japonicum. FAME profiles of these strains of $B$. japonicum were characterized by the quantitative variations in 16:1 $\omega 5$ cis, 17:0, 17:1 $\omega$ 8 cis and 19 cyclo $\omega 8$ cis and qualitative variations in 16:0 and summed feature 7 .

FAME analysis correctly distinguished the unknown strains of Bradyrhizobium sp. obtained from mungbean root nodules at both the species and subspecies. Among unknown 36 strains, each fatty acid group, FAG-I, FAG-II, FAG-III, FAG-IV and FAG-V were included $3,4,8,12$ and 8 strains, respectively. Twelve mungbean strains of Bradyrhizobium sp. belonging to FAG-IV showed a unique and specific fatty acid profiles at the composition of fatty acid 19:0 cyclow8cis. 


\section{Principal component analysis}

A two-dimensional plot of FAME principal components 1 and 2 is shown in Fig. 2. Two Bradyrhizobium species, B. japonicum (FAG-III and FAG-V) and B. elkanii (FAG-I and FAG-II) were quietly separated and distinguishable on this plot. However, the mungbean strains of Bradyrhizobium sp. (FAG-IV) were relatively close to $B$. japonicum but distinct to $B$. elkanii. FAME principal component analysis also revealed that Bradyrhizobium strains obtained from soybean and mungbean were close relationships within same FAGs but distinctly to different FAGs. It also revealed that Bradyrhizobium strains isolated from each prefecture within Kyushu area were not unclear relationships between FAGs and their locations (Data not shown).

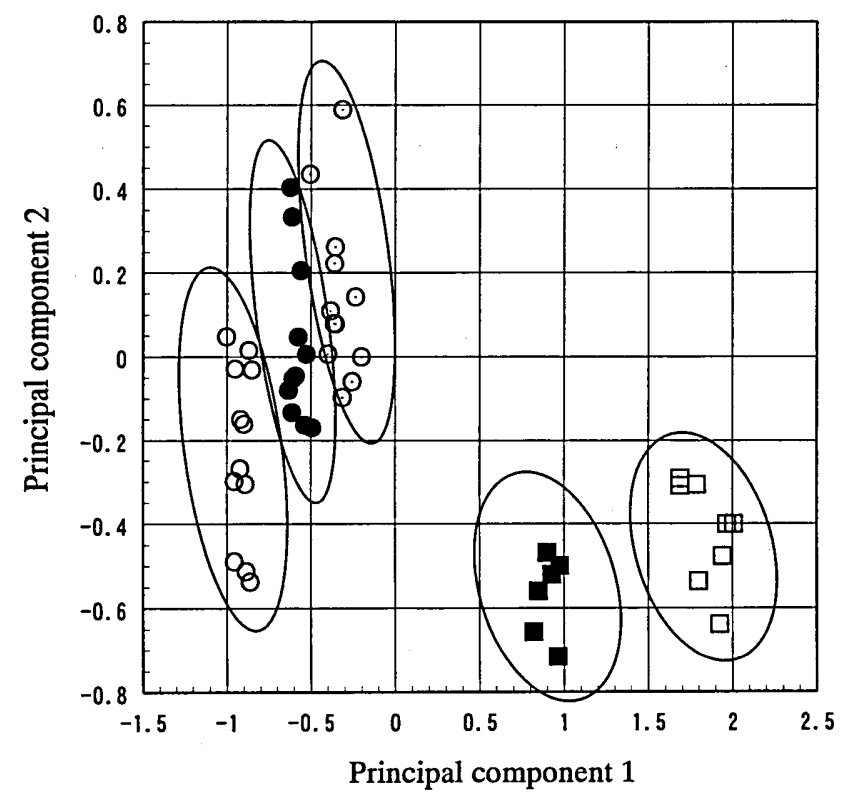

FAG-I

FAG-II

- FAG-III

$\odot$ FAG-IV

O FAG-V

Fig. 2. Two-dimensional plot of principal component analysis for 49 Bradyrhizobium strains. Samples are indicated as follows: $\mathbf{\square}$, FAG-I; $\square$, FAG-II;, FAG-III; $\odot$ FAG-IV; $\bigcirc$, FAG-V

\section{DISCUSSION}

In this experiment, FAME analysis was effectively characterized the unknown strains of Bradyrhizobium species obtained from mungbean root nodules. FAME analysis in this experiment also revealed the existence of subgroups and fatty acid phenotypes between MAFF strains of $B$. japonicum and $B$. elkanii isolated from soybean root nodules. Several recent research of the genus Bradyrhizobium also showed clear separation between $B$. japonicum and $B$. elkanii by cellular fatty acid analysis and reported 
the existence of one or more additional subgroups within these species. Graham et al. (1995) reported the existence of two subgroups in B. japonicum (Group IA and Group IB) and a single subgroup in $B$. elkanii. Tighe et al. (2000) reported existence of two subgroups in B. elkanii (Group I and II) and a single subgroup in B. japonicum. In this experiment, FAME analysis revealed the existence of different two subgroups each $B$. japonicum (FAG-III and FAG-V) and B. elkanii (FAG-I and FAG-II) strains consisting of MAFF strains (soybean) and Kyushu strains (mungbean). Comparing the data of fatty acid profiles presented in this experiment and previous reports, our results suggested the good agreement with these reports that are corresponded to $B$. japonicum Group IA and FAG-V, Group IB and FAG-III, B. elkanii Group I and FAG-I, Group II and FAG-II, respectively. Although unidentified strains of Bradyrhizobium sp. isolated from mungbean root nodule (FAG-IV) showed a unique and specific fatty acid profiles, similarity of fatty acid compositions were not observed by comparing the data of fatty acid compositions that were previously reported.

Identification and classification of Bradyrhizobium strains isolated from mungbean revealed significant fatty acid differences and designed as the distinct five groups, FAG-I to FAG-V. In this experiment, UPGMA clustering and FAME PCA plots showed that FAG-I and FAG-II strains had great relevance to B. elkanii, and FAG-III and FAG-V strains had close related to B. japonicum, respectively (Fig. 1. and Table 2.). However, these analyses also revealed unclear relationships between mungbean strains and their isolation locations within Kyushu area. Another taxonomic criteria based on species-specific PCR analysis and cultural characteristics with the mungbean strains of FAG-I to FAG-V were tested and obtained identical results with whole cellular fatty acid analysis (data not shown). Therefore, we concluded that FAME analysis provides a relatively simple and reliable procedure for the initial characterization of Bradyrhizobium strains.

The data presented here support the distinction between $B$. japonicum and $B$. elkanii and prove the close relationship to host plants of soybean and mungbean. Moreover mungbean strains belonging to FAG-IV showed a unique and specific phenotypes based on FAME analysis and considered as a now subgroups of Bradyrhizobium species. Because the data of fatty acid profiles for FAG-IV presented close relationship to FAG-III and FAG-V but obviously different to their subgroups. Several recent studies of the bardyrhizobia also showed clear separation between $B$. japonicum and B. elkanii (Dupuy et al., 1994; So et al., 1994; Gao et al., 1994) and reported the existence of one or more additional groups of bardyrhizobia. The need for additional work on identification and classification of Bradyrhizobium species is evident in a number of different studies and will necessitate the evaluation of strains from many different plant legumes or soils. For this reason, we make no attempt to provide species designation for the new groups of Bradyrhizobium sp. isolated from mungbean. Our research is prefer to lead a more detailed and polyphasic approach using a more diverse group of bardyrhizobia including strains from soybean, mungbean and more different crops and pasture legumes.

\section{ACKNOWLEDGEMENT}

This experiment was supported in part by the Special Coordination Funds for Promoting Science and Technology of the Science and Technology Agency of the 
Japanese Government.

\section{REFERENCES}

Bottomley, P. J., C. Hsin-Hua and S. R. Strain 1994 Genetics structure and symbiotic characteristics of a Bradyrhizobium population recovered from a pasture soil. Appl. Environ. Microbiol., 60: 1754-1761

Durpy, N., A. Willems, D. Pot, B. Dewettinck, L. Vandenbruaene, G. maestrojuan, B. Dreyfus, K. Kersters, M. D. Collins and M. Gillis 1994 Phenotypic and genotypic characterization of bardyrhizobia nodulating the leguminous tree Acacia albida. Int. J. Syst. Bacteriol., 44: 461-473

Gao, J. L., J. G. Sun, Y. li, E. T. Wang and W. X. Chen 1994 Numerical taxonomy and DNA relatedness of tropical rhizobia isolated from Hainan province, Chaina. Int. J. Syst. Bacteriol. 44: 151-158

Graham, P. H., M. J. Sadowsky, S. W. Tighe, J. A. Thompson, R. A. Date, J. G. Howieson and Thomas, R. 1995 Differences among strains of Bradyrhizobium in fatty acid-methyl ester analysis. Canadian Journal of Microbiology, 41: 1038-1042

Hollis, A. B., W. E. Kloos G. E. and Elkan 1981 DNA:DNA hybridization studies of Rhizobium japonicum and related Rhizobiaceae. J. Gen. Microbiol., 123: 215-222

Jarvis, B. D. W. and S. W. Tighe 1994 Rapid identification of Rhizobium species based on cellular fatty acid analysis. Plant Soil, 161: $31-41$

Jordan, D. C. 1982 Transfer of Rhizobium japonicum Buchanan 1980 to Bradyrhizobium gen. nov., a genus of slow-growing, root nodule bacteria from leguminous plants. Int. J. Syst. Bacteriol., 32: 136-139

Kuykendall, L. D., B. Saxena, T. E. Devine and S. E. Udell 1992 Genetic diversity in Bradyrhizobium elkanii sp. nov. Can J. Microbiol., 38: 501-505

Sasser, M. 1990 Identification of bacteria by gas chromatography of cellular fatty acids, MIDI Technical Note 101 Microbial ID, Inc., Newark, DE, USA

So, R. B., J. K. Ladha and J. P. W. Young 1994 Photosynthetic symbionts of Aeschynomene spp form a cluster with bardyrhizobia on the basis of fatty acid and rRNA analyses. Int. J. Syst. Bacteril. 44: $392-403$

Somasegaram, P. and H. J. Hoben 1994 Handbook for Rhizobia. Methods in Legume Rhizobium Technology. Springer Verlag.

Tighe, S. W., P. de Lajudie, K. Dipietro, K. Lindström, G. Nick and D. B. W. Jarvis 2000 Analysis of cellular fatty acids and phenotypic relationships of Agrobacterium, Bradyrhizobium, Mesorhizobium, Rhizobium and Sinorhizobium species using the sherlock microbial identification system. Int. J. Syst. Evol. Microbiol, 50: 787-801

Urts, B. E. and G. H. Elkan 1996 Genetic diversity among Bradyrhizobium isolates that effectively nodulate peanut (Arachis hypogeae). Can. J. Microbiol., 42: 1121-1130

Xu, L. M., C. Ge, Z. Cui, J. Li and H. Fan, 1995 Bradyrhizobium liaoningense sp. nov., isolated from the root nodules of soybeans. Int. J. Syst. Bacteriol, 45: 706-711

Yokoyama, T., S. Ando, T. Murakami and H. Imai 1996 Genetic variability of the common nod gene in soybean bradyrhizobia isolated in Thailand and Japan. Can. J. Microbiol., 42: 1209-1218

Young, J. P. W. and K. E. Haukka 1996 Diversity and phylogeny of rhizobia. New Phytol, 133: 87-94

Zhang, X., G. Nick, S. Kaijalainen, Z. Terefework, L. Paulin, S. W. Tighe, P. H. Graham and K. Lindstr_m 1999 Phylogeny and diversity of Bradyrhizobium strains isolated from the root nodules of peanut (Arachis hypogaea) in Sichuan, China. Syst Appl Microbiol, 22: 378-386

van Rossum D., F. P. Schuurmans, M. Gillis, A. Muyotcha, H. W. van Verseveld, A. H. Stouthamer and F. C. Boogerd 1995 Genetic and phonetic analyses of Bradyrhizobium strains nodulating peanut (Arachis hypogaea L.) root. Appl. Environ. Microbiol., 61: 1599-1609 